\title{
Association of Retinopathy with Chronic Kidney Disease in Diabetes Mellitus
}

\author{
Rouf RSB ${ }^{\mathrm{a}}$, Ashrafuzzaman $\mathrm{SM}^{\mathrm{b}}$, Latif $\mathrm{ZA}^{\mathrm{c}}$
}

\begin{abstract}
Background: Diabetic retinopathy (DR) and nephropathy are two major complications of diabetes mellitus carrying significant morbidity and mortality. In this study DR was investigated in different stages of chronic kidney disease (CKD) to find out possible association of these two devastating complications.

Methods: This cross-sectional study was conducted in 150 diabetic patients having CKD in BIRDEM. CKD was defined as estimated glomerular filtration rate (eGFR) of $<60 \mathrm{ml} / \mathrm{min} / 1.73 \mathrm{~m}^{2}$ and/or urinary albumin excretion rate $(U A E R)>30 \mathrm{mg} /$ day in at least two occasions in 3 months apart. Retinopathy was assessed by direct fundoscopic examination and confirmed by color fundus photography. Severe DR (SDR) included proliferative diabetic retinopathy, severe non-proliferative $D R$ and maculopathy; whereas microaneurysm regarded as non-severe retinopathy.
\end{abstract}

Results: Majority (68\%) of the respondents had some form of retinopathy (38.35\% SDR and 29.65\% nonsevere). There was strong association between different levels of albuminuria (UAER) and DR $(p<0.0001)$. On the contrary $D R$ did not correspond with stages of CKD $(P=0.349)$. Hypertension (79.5\%) and dyslipidaemia (59\%) were common co-morbidities.

Conclusion: This study concluded that DR prevalence was more in nephropathy along with significant association with UAER. Whereas different stages of CKD was not associated with stages of DR. This finding focused the necessity of regular retinal examination irrespective of the stage of renal involvement.

Key words: Diabetic Retinopathy(DR), Chronic Kidney disease(CKD)

(BIRDEM Med J 2018; 8(3): 210)

\section{Introduction}

Diabetes mellitus (DM) is one of the most common noncommunicable diseases affects around 120 million people worldwide. The late complications of DM result in reduced life expectancy and bear major health costs. The complications of DM are due to both microvascular and macrovascular damage. ${ }^{1}$ The metabolic and haemodynamic disturbances in Diabetes result in

\section{Author Information}

a. Dr. Rushda Sharmin Binte Rouf, FCPS (Medicine) MD (EM), Registrar, Department of Endocrinology, BIRDEM

b. Prof. S. M. Ashrafuzzaman , MD (EM), DEM, Professor, Department of Endocrinology, BIRDEM

c. Prof. Zafar Ahmed Latif, FCPS (Medicine), Director General, BIRDEM

Address of Correspondence: Dr. Rushda Sharmin Binte Rouf, FCPS (Medicine) MD (EM), Registrar, Department of Endocrinology, BIRDEM, Dhaka. E- mail: rushdalubna767@, gmail.com

Received: November 26, 2017

Accepted: June 30, 2018 increased vessel permeability, raised blood pressure, and altered regulation of intracapillary pressure. This microvascular complication is specific to diabetes where small blood vessels throughout the body are affected but the disease process is of particular danger in three sites: retina, renal glomerulus and nerve sheaths. In the kidney, these changes may lead to increased trafficking of plasma proteins across the glomerular membrane and to the appearance of protein in the urine. The strong relationship between proteinuria and a constellation of other diabetic complications supports the view that elevated urinary protein excretion reflects a generalized vascular disturbance. Since retinal and renal vessels are exposed to the diabetic milieu, it is often assumed that progression of diabetic retinopathy and nephropathy occurs at the same time. ${ }^{2}$

Several studies have been done to find out the correlation between these two complications as they share common 
etiological factors. ${ }^{3,4,5}$ Significant correlation between retinal and glomerular disease in diabetes were found based on fundoscopic findings with histopathological changes in kidney in type 1 patients. ${ }^{3}$ Another study showed that $82 \%$ patients with overt proteinuria had retinopathy. ${ }^{4}$ Estacio et al aimed to find out overt proteinuria as a predictor of retinopathy previously. ${ }^{5}$

Despite the catastrophic effects of diabetic retinopathy on vision and quality of life, the predictive value of this opthalmological complication for development of chronic kidney disease(CKD) or worsening renal function or vice versa is not fully defined across the spectrum of kidney disease in diabetes.

Bangladesh has the 10th largest number of diabetic patients in the world. ${ }^{6}$ Prevention of complication should be emphasized more to reduce the health burden. In this regard we investigated retinopathy in different stages of CKD to find out possible association in the progression of these two devastating complications with an aim to improve quality of life in terms of morbidity and mortality in diabetes.

\section{Methods}

This cross-sectional study was conducted in 150 diabetic patients having CKD in BIRDEM. CKD was defined as estimated glomerular filtration rate (eGFR) of $<60\left(\mathrm{ml} / \mathrm{min} / 1.73 \mathrm{~m}^{2}\right)$ and/or urinary albumin excretion rate $($ UAER $)>30 \mathrm{mg} /$ day in at least two occasions in 3 months apart. Retinopathy was assessed by direct fundoscopic examination and confirmed by color fundus photography. Severe DR (SDR) included proliferative diabetic retinopathy, severe non-proliferative DR and maculopathy whereas microaneurysm regarded as nonsevere retinopathy.

\section{Results}

Total patients were 150 with mean age of $45 \pm 10.3$ years. The mean duration of DM was $9.4 \pm 6.7$. (Table I) Among the co-morbidities HTN was common in the population $(79.5 \%)$,dyslipidaemia (59\%), ischemic heart disease $(43.6 \%)$ and stroke (12.8\%) were other co morbid conditions found among the study group (Fig 1). Among 150 respondents, $102(68 \%)$ had some form of retinopathy. Among the $68 \%$ of retinopathy, severe diabetic retinopathy (SDR)(PDR, severe NPDR, maculopathy) was present in $38.35 \%$ and $29.65 \%$ were having non severe retinopathy(NDR). NPDR was
$45.5 \%$, severe NPDR was $18.2 \%$, PDR was $31.8 \%$, and maculopathy was $4.5 \%$ (Fig - 2). The association of 24 hours urinary total protein (24h UTP) and frequency of retinopathy was assessed (Table II). In microalbuminuric (albuminuria $<300 \mathrm{mg} /$ day)stage only $36 \%$ had retinopathy. Whereas in overt proteinuric ( 0.3 to $3.5 \mathrm{gm} /$ day) and nephrotic stage ( $>3.5 \mathrm{gm} /$ day) it rose to $86.48 \%$ and $76.93 \%$ respectively with significant association $(\mathrm{p}<0.05)$. The association between eGFR and retinopathy was unremarkable $(p>0.05)$. The frequency of retinopathy in different stages of CKD was variable. The frequency was $73.34 \%$ in stage 3 and $67.86 \%$ and $58.83 \%$ in stage 4 and 5 respectively (Table III)

Table I Baseline characteristics of study population (N-150)

\begin{tabular}{lcc}
\hline Parameters & $\mathrm{n}(\%)$ & Mean $\pm \mathrm{SD}$ \\
\hline Age (years) & & \\
$<50$ & $44(29.3 \%)$ & $45( \pm 10.3)$ \\
$\geq 50$ & $106(70.6 \%)$ &
\end{tabular}

Gender

Male $\quad 80(53.33 \%)$

Female $\quad 70(46.66 \%)$

Duration of DM (years)

$\begin{array}{ll}<5 & 34(22.66 \%)\end{array} \quad 9.4( \pm 6.7)$

5-10 62(41.33\%)

$>10 \quad 54(36 \%)$

Glycemic status

HbAlc (\%)

$\begin{array}{lll}<7 & 30(20 \%) & 9.2( \pm 5.8)\end{array}$

$>7 \quad 120(80 \%)$

Area of residence

Urban

103(68.66\%)

Rural

$47(31.33 \%)$

Treatment

Only OAD

$40(26.66 \%)$

Insulin $\pm \mathrm{OAD}$

$110(73.3 \%)$

$[\mathrm{OAD}=$ Oral anti diabetic drugs $]$ 


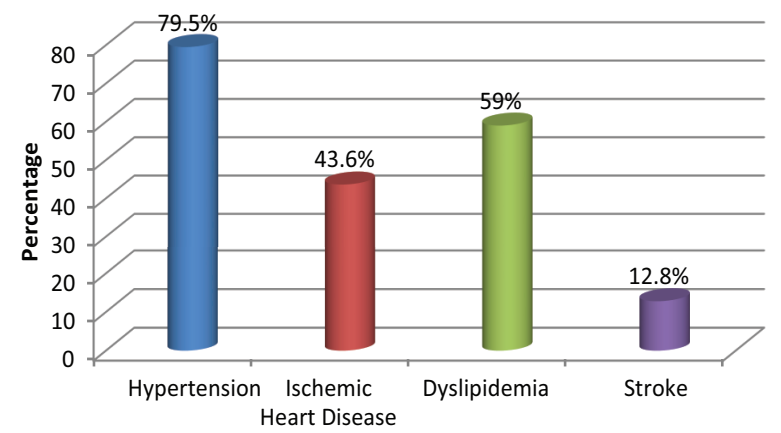

Co-morbidities

Figure 1 Co-morbidities among the study subjects $(\mathrm{N}=150)$

Among the 150 respondents 58(38.35\%) had severe diabetic retinopathy (Severe DR), 44 (29.65\%) had non severe diabetic retinopathy (Non severe DR) and only $48(32 \%)$ had no retinopathy(No DR).

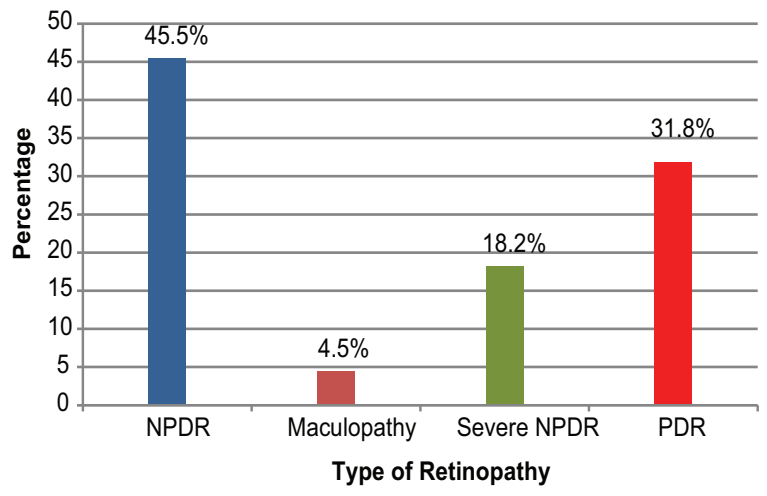

Figure 2 Type of retinopathy among the study subjects $(\mathrm{N}=150)$

Table II Association of 24 hours urinary total protein with retinopathy

\begin{tabular}{lcccc}
\hline UTP $($ gm & DR & No DR & Total & $\begin{array}{c}\text { P } \\
\text { value }\end{array}$ \\
/24 hours $)$ & $\mathrm{n}(\%)$ & $\mathrm{n}(\%)$ & & 0.00001 \\
\hline $0.03-0.3$ & $32(64 \%)$ & $18(36 \%)$ & $50(100 \%)$ & 0.00 \\
$0.3-3.5$ & $10(13.52 \%)$ & $64(86.48 \%)$ & $74(100 \%)$ & \\
$>3.5$ & $6(23.07 \%)$ & $20(76.93 \%)$ & $26(100 \%)$ & \\
Total & $48(32 \%)$ & $102(68 \%)$ & $150(100 \%)$ & \\
\hline
\end{tabular}

Pearson Chi-Square value $=36.1028$
Table III Association between eGFR and retinopathy

\begin{tabular}{lccc}
\hline eGFR & No retinopathy & DR & Total \\
\hline $30-59$ & $16(26.66 \%)$ & $44(73.34 \%)$ & $60(100.0)$ \\
$15-29$ & $18(32.14 \%)$ & $38(67.86 \%)$ & $56(100.0)$ \\
$<15$ & $14(41.17 \%)$ & $20(58.83 \%)$ & $34(100.0)$ \\
\hline Total & $48(32 \%) 102(68 \%)$ & $150(100.0)$ \\
\hline
\end{tabular}

Pearson Chi-Square value $=2.1006 . \quad \mathrm{P}=0.349836$

\section{Discussion}

This cross-sectional study was carried out to observe the correlation between diabetic retinopathy in different stages of CKD in DM. It was found that among the 150 patients $68 \%$ patients had some form of diabetic retinopathy. More than half (56.4\%) were suffering from vision threatening severe form of retinopathy. The result is in agreement with a study conducted by Grunwald on 925 participants with DM. Out of 925 subjects, $456(49 \%)$ had diabetic retinopathy. ${ }^{7}$ In a recent study conducted by Wong et al, the prevalence of diabetic retinopathy in patients with CKD due to DM was shown to be $34.7 \%$ after adjusting for various co-morbidities. ${ }^{8}$

Our study found proteinuria as a strong predictor of retinopathy. Several studies supported this evidence. Cruickshanks et al, carried out a prospective study on 1139 diabetic patients who did not have hematuria or a history of renal disease, to find out the association between albuminuria and diabetic retinopathy. After a mean follow up period of 4 years, it was found that participants with microalbuminuria were 1.7 to 3.2 times as likely to have retinopathy as those without microalbuminuria, in univariate analyses. This relationship remained after controlling for other potential confounders such as glycemia, hypertension, smoking and duration of diabetes. ${ }^{9}$ Manaviat et al studied 590 type 2 diabetes patients for retinopathy and albuminuria and found that there was significant association between them.${ }^{10}$ Vigstrup et al, conducted a prospective cohort study on 43 patients and concluded that that even a slightly raised UAE (Urinary Albumin Excretion) is a strong predictor with respect to development of proliferative diabetic retinopathy, as well as nephropathy. Their study recommends using UAE to select those patients needing a closer control 
and follow up. ${ }^{11}$ In the above-mentioned studies albuminuria had been considered as a risk marker of diabetic retinopathy. Thus excretion of albumin in urine can be regarded as a sign of kidney involvement and can reflect generalized vessel damage throughout the body.

On the contrary this study found no association of retinopathy in different stages of CKD. We concluded though DR was more prevalent in nephropathy, the progression of these two target organ damage may not coincide. In CRIC study baseline unadjusted analysis showed significant association between retinopathy and CKD suggesting retinovascular pathology as a reflection of disease of other vascular beds, including kidneys. But in subsequent adjusted multivariate analysis the association of these two waned. They concluded presence and severity of DR may not provide any additional prognostic information regarding risk of CKD progression and vice versa which is similar with the current study. ${ }^{12}$ Previously same observation was found in different studies. Goldstein et al showed that a decline in renal function was not followed by the same decline in retinopathy. ${ }^{13}$ Some also found that rapid decline in renal function with or without significant proteinuria were inconsistent with natural history of DR. Some patients with advanced retinopathy had little or no renal disease. They emphasized on regular fundoscopic screening particularly in presence of proteinuria irrespective of stages of nephropathy which is consistent to this study. ${ }^{13-15}$ Among the co morbidities, HTN and dyslipidaemia were common. It has been observed that hypertension independent of hyperglycaemia up regulates the vascular endothelial growth factor expression in retinal endothelial cells and ocular fluids and increase the risk of developing CKD than those without hypertension. Another study also found that dislipidemia plays a major role in developing complications of DM. Patients with combined dislipidemia have an increased incidence of retinal abnormalities similar to the present study. ${ }^{16,17}$

Hbalc was found more than $7 \%$ in $80 \%$ respondents in this study. Manaviat et al, found that HbAlc has got significant association with the occurrence of retinopathy. ${ }^{10}$ Stratton et al, found that there was a highly significant positive association of $\mathrm{HbAlc}$ with incidence and progression of retinopathy in both univariate and multivariate analysis. ${ }^{12}$ Wong et al also found similar results. ${ }^{15}$ Our study showed that the frequency of retinopathy is more in the age group of $>50$ years. That age is a risk factor for retinopathy as well as other vascular complications of diabetes is a well known fact. Manaviat et al found in their study that increasing age is significantly associated with the development of diabetic retinopathy $(\mathrm{P}=0.014) .{ }^{10}$ Sobngwi et al found that diabetic retinopathy is significantly associated with age $(\mathrm{P}<0.001) .{ }^{18}$ Mean duration of DM was $9.4( \pm 6.7)$ in our study. Previous studies have demonstrated that both hypertension and duration of diabetes were independent risk factors for diabetic retinopathy. Yau et al found that the prevalence of any diabetic retinopathy increased with diabetes duration, HbAlc and blood pressure. ${ }^{19}$ Thus we found aging, longer duration of DM are non modifiable risk factors whereas HTN and poor glycemic status are modifiable risk factors for development of both retinopathy and nephropathy in DM.

\section{Conclusion}

In this study diabetic retinopathy was more prevalent in nephropathy irrespective of stages of renal failure. Proteinuria is a significant predictor for development of retinopathy. This finding focused the necessity of regular retinal examination in diabetic patients particularly who has any form of albuminuria to protect vision, thus improving the quality of life in diabetic patients.

Conflict of interest: Nothing to declare.

\section{References}

1. Gale EAM, Anderson JV. Diabetes mellitus and other disorders of metabolism. In: Kumar, P, Clark, M (eds.) Kumar and Clark's Clinical Medicine.7th ed,UK, Saunders Ltd; 2009. p. 1030-1075.

2. Perna A, Remuzzi G. Abnormal permeability to proteins and glomerular lesions: A meta-analysis of experimental and human studies. Am J Kidney Dis 1996; 27: 34-41.

3. Chavers BM, Mauer SM, Ramsay RC, Steffes MW. Relationship between retinal and glomerular lesions in IDDM patients. Diabetes Care 1994; 43: 441-46.

4. Lee KU, Park JY, Kim SW, Lee MII, Kim GS, Park S-K, et al. Prevalence and associated features of albuminuria in Koreans with NIDDM. Diabetes Care 1995; 18:793-99.

5. Estacio RO, McFarling E, Biggerstaff S, Jeffers BW, Johnson D, Schrier RW,et al. Overt albuminuria predicts diabetic retinopathy in Hispanics with NIDDM. American Journal of Kidney Disease 1994; 31: 947-53. 
6. Akhter A, Fatema k, Afroz A, Ali 1, Hussain A. Prevalence of diabetes mellitus and associated risk indicators in a rural Bangladeshi population. The open diabetes journal 2011; 4 : 6-11.

7. Grunwald JE, Alexander J, Ying JS, Richard L, Dina H A, Shawn B, et al. Retinopathy and Chronic Kidney Disease in the Chronic Renal Insufficiency Cohort (CRIe) Study. Arch Ophthalmo1 2012; 130: 1136-44.

8. Wong CW, Lamoureux EL, Cheng CY, Cheung G, Cheng CY, Tai ES, et al. Increased Burden of Vision Impairment and Eye Diseases in Persons with Chronic Kidney Disease - A Population - Based Study. E Bio Medicine 2016; 5: 193-97.

9. Cruickshanks KJ, Ritter LL, Klein R. The Association of Microalbuminuria with Diabetic Retinopathy: The Wisconsin Epidemiologic Study of Diabetic Retinopathy. Ophthalmology 1993; 100: 862-67.

10. Manaviat MR, Afkhami M, Shoja MR. Retinopathy and microalbuminuria in type II diabetic patients. BMC Ophthalmol. 2004; 4:9.

11. Vigstrup J, Mogensen CE. Proliferative diabetic retinopathy: at risk patients identified by early detection of microalbuminuria. Acta Ophthalmologica 1985; 63: 530-34.

12. Grunwald JE, Pistilli M, Shuang GY,Daniel E,Maguire MG,Xie D, et al. Retinopathy and progression of CKD : The CRIC Study.Clin J Am Soc Nephrol 2014;9:1217-24.
13. Goldstein M, Blinder KJ, Wilson RJ,Weidmeyer HM,Little MR,England JD, et al. Glycaemic control and development of retinopathy in youth-onset insulin-dependent Diabetes Mellitus. Ophthalmology 1993; 100: 1125-31.

14. Prokash J, Lodha M. Retinopathy and other microvascular complications. Diabetic Care 2007; 16: 23-27.

15. Veechi ML, Fuiano G, Francesco M, Mancuso D,Faqa T,Sponton A,et al. Prevalence and severity of anaemia in patients with Type 2 Diabetic nephropathy and different degrees of chronic renal insufficiency. Nephron clin prac 2007; 105, 62-67.

16. Coresh J, Byrd D, Astor BC, Briggs JP,Eggers PW,Lacher DA,et al. Chronic kidney disease awareness, prevalence, and trends among U.S. adults. Journal of American Society of Nephrology 2005; 16: 180 -88.

17. Dodson PM, Carlton PJ. Retinal vascular abnormalities in hyperlipidemia. Trans Opthal Soc UK 2004; 101:17-21.

18. Sobngwi E, Mbanya J, Moukouri EN, Ngu KB. Microalbuminuria and retinopathy in a diabetic population of Cameroon. Diabetes Research and Clinical Practice 1999; 44: $191-96$

19. Yau JW, Rogers SL, Kawasaki R, Lamoureux EL,Kowalski JW,Bek T,et al. Meta-Analysis for Eye Disease (META-EYE) Study Group. Global prevalence and major risk factors of diabetic retinopathy Diabetes Care 2012; 35:556-64. 